PREPARED FOR THE U.S. DEPARTMENT OF ENERGY, UNDER CONTRACT DE-AC02-76CH03073

PPPL-3823

PPPL-3823

UC-70

Multi-grid Particle-in-cell Simulations

of Plasma Microturbulence

by

J.L.V. Lewandowski

June 2003

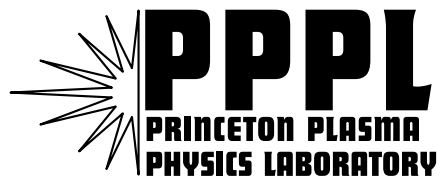

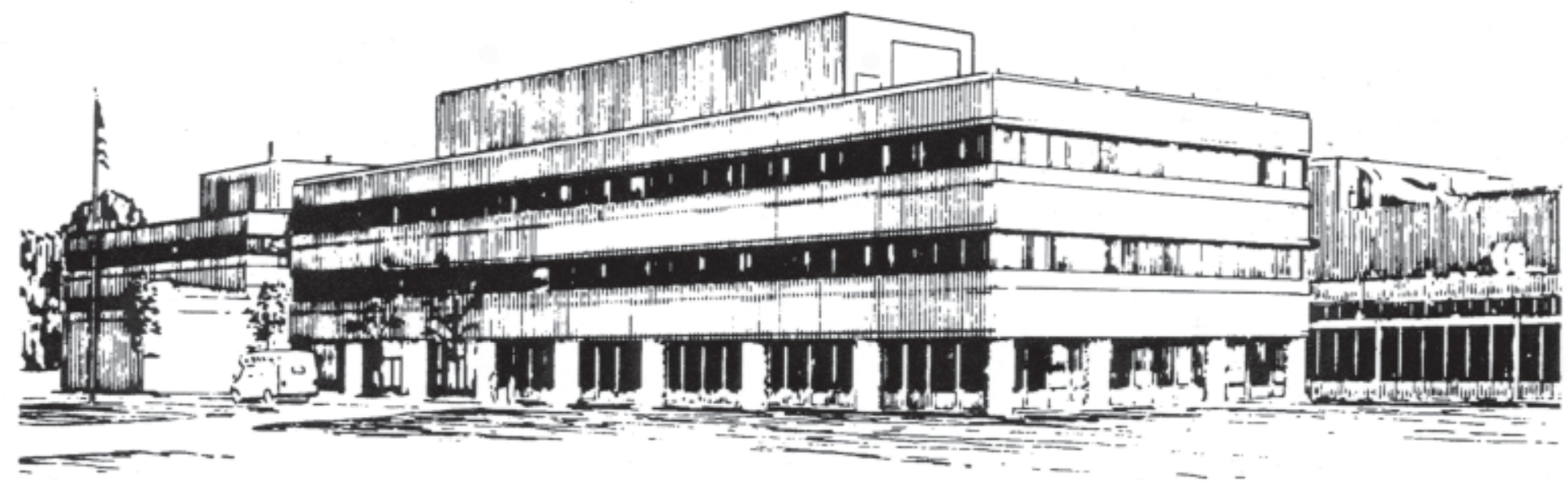

PRINCETON PLASMA PHYSICS LABORATORY PRINCETON UNIVERSITY, PRINCETON, NEW JERSEY 


\section{PPPL Reports Disclaimer}

This report was prepared as an account of work sponsored by an agency of the United States Government. Neither the United States Government nor any agency thereof, nor any of their employees, makes any warranty, express or implied, or assumes any legal liability or responsibility for the accuracy, completeness, or usefulness of any information, apparatus, product, or process disclosed, or represents that its use would not infringe privately owned rights. Reference herein to any specific commercial product, process, or service by trade name, trademark, manufacturer, or otherwise, does not necessarily constitute or imply its endorsement, recommendation, or favoring by the United States Government or any agency thereof. The views and opinions of authors expressed herein do not necessarily state or reflect those of the United States Government or any agency thereof.

\section{Availability}

This report is posted on the U.S. Department of Energy's Princeton Plasma Physics Laboratory Publications and Reports web site in Fiscal Year 2003. The home page for PPPL Reports and Publications is: http://www.pppl.gov/pub_report/

DOE and DOE Contractors can obtain copies of this report from:

U.S. Department of Energy

Office of Scientific and Technical Information

DOE Technical Information Services (DTIS)

P.O. Box 62

Oak Ridge, TN 37831

Telephone: (865) 576-8401

Fax: (865) 576-5728

Email: reports@adonis.osti.gov

This report is available to the general public from:

National Technical Information Service

U.S. Department of Commerce

5285 Port Royal Road

Springfield, VA 22161

Telephone: $1-800-553-6847$ or

(703) $605-6000$

Fax: (703) 321-8547

Internet: http://www.ntis.gov/ordering.htm 


\title{
Multigrid particle-in-cell simulations of plasma microturbulence
}

\author{
J.L.V. Lewandowski \\ Princeton Plasma Physics Laboratory \\ Princeton University, P.O. Box 451, Princeton NJ 08543
}

(June 9, 2003)

\begin{abstract}
A new scheme to accurately retain kinetic electron effects in particle-in-cell (PIC) simulations for the case of electrostatic drift waves is presented. The splitting scheme, which is based on exact separation between adiabatic and nonadiabatic electron responses, is shown to yield more accurate linear growth rates than the standard $\delta f$ scheme. The linear and nonlinear elliptic problems that arise in the splitting scheme are solved using a multigrid solver. The multigrid particle-in-cell approach offers an attractive path, both from the physics and numerical points of view, to simulate kinetic electron dynamics in global toroidal plasmas.
\end{abstract}

Pacs \# : 52.35Kt, 52.30Jb, 52.35Ra 


\section{INTRODUCTION}

It is now generally accepted in the fusion community that low-frequency, small-scale instabilities (eg. drift waves, Ion Temperature Gradient-driven (ITG) modes) are major contenders for the anomalous, cross-field transport observed in tokamaks ${ }^{1}$ and stellarators ${ }^{2}$. The impact of electron dynamics on linear modes and fully developed turbulence is a most important subject.

The treatment of electrons in particle-in-cell simulations is made difficult due to the

fact that the electrons move $\sim \sqrt{m_{i} / m_{e}}$ times faster than the ions. However, for drifttype $\operatorname{modes}^{3,4}$, the bulk of the electrons respond adiabatically to the waves and it may be advantageous to focus on the nonadiabatic part of the electron response.

The paper is organized as follows; in section II, the governing equations for the (standard) $\delta f$ scheme are given. The splitting scheme is presented in section III. A consequence of the splitting scheme is that, in addition to the usual gyrokinetic Poisson equation, a nonlinear elliptic equation is to be solved. This problem is addressed in section IV where a multigrid Poisson solver is described and tested. Numerical experiments comparing the standard $\delta f$ scheme and the splitting scheme are reported in section V. Concluding remarks are presented in section VI.

\section{STANDARD $\delta F$ SCHEME}

In this section, we present the model equations governing electrostatic drift waves in shearless slab geometry based on the standard $\delta f$ scheme $^{5}$. The magnetic field is taken to be of the form $\mathbf{B}_{0}=B_{0}(\widehat{\mathbf{z}}+\theta \widehat{\mathbf{y}})$ where $\theta \ll 1$ is a small parameter related to the tilt of the magnetic field lines with respect to the $z$ direction; the equilibrium direction is taken to be along the $x$ axis. Our starting equation is the collisionless, electrostatic, gyrokinetic Vlasov equation, in the long-wavelength limit, for particle species $j$ with mass $m_{j}$ and charge $q_{j}$

$$
\frac{d F_{j}}{d t} \equiv \frac{\partial F_{j}}{\partial t}+\left(v_{\|} \widehat{\mathbf{b}}_{0}+\mathbf{V}_{E}\right) \cdot \nabla F_{j}-\frac{q_{j}}{m_{j}} \widehat{\mathbf{b}}_{0} \cdot \nabla \Phi \frac{\partial F_{j}}{\partial v_{\|}}=0
$$


where $\widehat{\mathbf{b}}_{0}=\mathbf{B}_{0} / B_{0}$ a unit vector and $\mathbf{V}_{E}=c \widehat{\mathbf{b}}_{0} \times \nabla \Phi / B_{0}$ is the $\mathbf{E} \times \mathbf{B}$ drift velocity. The standard $\delta f$ scheme is based on the representation

$$
F_{j}=F_{M j}+\delta f_{j}
$$

where $F_{M j}$ is the Maxwellian distribution. Introducing the marker weight, $W_{j} \equiv \delta f_{j} / F_{j}$, one obtains

$$
\frac{d W_{j}}{d t}=\left(1-W_{j}\right)\left(\mathbf{V}_{E} \cdot \boldsymbol{\kappa}_{j}-\frac{q_{j}}{T_{j}} v_{\|} \nabla_{\|} \Phi\right)
$$

where $\boldsymbol{\kappa}_{j}=\boldsymbol{\kappa}\left[1-\frac{\eta_{j}}{2}\left(1-\bar{v}_{\|}^{2}\right)\right], \bar{v}_{\|}=v_{\|} / V_{t h j}$ and $\boldsymbol{\kappa}=-\boldsymbol{\nabla} n_{0} / n_{0}$. In gyrokinetic units $\left(\omega_{c i} t \mapsto t ; v_{\|} / c_{s} \mapsto v_{\|} ; \rho_{s} \kappa \mapsto \kappa ; \rho_{s} \nabla \mapsto \nabla ; e \Phi / T_{e} \mapsto \Phi\right)$, the equations of motion are

$$
\begin{gathered}
\frac{d \mathbf{r}}{d t}=v_{\|} \widehat{\mathbf{b}}_{0}+\widehat{\mathbf{b}}_{0} \times \nabla \Phi \\
\frac{d v_{\|}}{d t}=-Z_{j} \frac{m_{i}}{m_{j}} \nabla_{\|} \Phi
\end{gathered}
$$

and the weight equation becomes

$$
\frac{d W_{j}}{d t}=\left(1-W_{j}\right)\left[\left(\widehat{\mathbf{b}}_{0} \times \nabla \Phi\right) \cdot \boldsymbol{\kappa}_{j}-\sigma_{j} v_{\|} \nabla_{\|} \Phi\right]
$$

where $\sigma_{j}=Z_{j} T_{e} / T_{j}$. The system of equations is closed with the gyrokinetic Poisson equation (in the long wavelength limit)

$$
\nabla_{\perp}^{2} \Phi=\int_{-\infty}^{+\infty}\left(\delta f_{e}-\delta f_{i}\right) d v_{\|}
$$

As shown in section $\mathrm{V}$, the linearized form of the standard $\delta f$ scheme, based on Eqs.(4-7), does not yield accurate growth rates, especially when the drive is small (small $\kappa$ ). For lowfrequency drift-type modes, only the slow electrons interact with the wave; the majority of the electrons does not 'see' the wave, that is they respond adiabatically. In order to address this accuracy problem, we introduce the splitting scheme in the next section. Numerical experiments presented in section $\mathrm{V}$ confirm that the splitting scheme yields accurate linear growth rates, even when few electrons are used in simulations. 


\section{SPLITTING SCHEME}

In order to address the accuracy problem mentioned in the previous section, we write the distribution $F_{j}$ as

$$
F_{j}=H(\Phi) F_{M j}+h_{j}
$$

where, as before, $F_{M j}$ is the Maxwellian distribution for particle species $j$ and $H(\Phi)$ is a yet undetermined function of the electrostatic potential. The so-called nonadiabatic part of the distribution function, $h_{j}$, is governed by

$$
\frac{d h_{j}}{d t}=-H \underbrace{\mathbf{V}_{E} \cdot \boldsymbol{\nabla} F_{M j}}_{\mathbf{1}}-v_{\|} \nabla_{\|} \Phi \underbrace{\left(H^{\prime}+\frac{q_{j}}{T_{j}} H\right)}_{\mathbf{2}} F_{M j}-\underbrace{H^{\prime} F_{M j} \frac{\partial \Phi}{\partial t}}_{\mathbf{3}},
$$

where a prime denotes a derivative with respect to $\Phi$ and the relation $\mathbf{V}_{E} \cdot \boldsymbol{\nabla} \Phi=0$ has been used. Term 1 represents the drive from the free energy whereas term 2 accounts for the parallel free streaming and the parallel force. Demanding that term $\mathbf{2}$ vanishes, we get

$$
\frac{d H}{d \Phi}=-\frac{q_{j}}{T_{j}} H
$$

with solution

$$
H=H_{0} \exp \left(-\frac{q_{j}}{T_{j}} \Phi\right)
$$

In the absence of turbulence, $\Phi=h_{j}=0$, we have $F_{j}=F_{M j}$ [Eq.(8)] so that $H_{0}=1$. A convenient splitting scheme for Eq.(1) is thus given by

$$
F_{j}=\exp \left(-\frac{q_{j}}{T_{j}} \Phi\right) F_{M j}+h_{j} .
$$

The equation governing the weight associated with the nonadiabatic part of the distribution function, $\overline{W_{j}} \equiv h_{j} / F_{j}$, can be easily derived using Eqs. $(1,11)$

$$
\frac{d \overline{W_{j}}}{d t}=\left(1-\overline{W_{j}}\right)\left(\mathbf{V}_{E} \cdot \boldsymbol{\kappa}_{j}+\frac{q_{j}}{T_{j}} \varphi\right)
$$

where $\varphi \equiv \partial \Phi / \partial t$. By construction, the contribution due to the free streaming markers has been removed from the weight equation [compare with the corresponding weight equation 
for the standard $\delta f$ scheme, Eq.(6)]. Representation (9) implies the computation of an additional scalar field, $\varphi$. The equation governing $\varphi$ can be obtained as follows. Multiply Eq.(1) by $v_{\|}^{k}$ and integrate over velocity space; the evolution equation for the velocity moment of order $k$

$$
M_{j}^{(k)} \equiv \int_{-\infty}^{+\infty} v_{\|}^{k} F_{j} d v_{\|} \quad(k=0,1,2, \cdots)
$$

can be written as

$$
\frac{\partial}{\partial t} M_{j}^{(k)}=-\mathbf{V}_{E} \cdot \nabla M_{j}^{(k)}-\widehat{\mathbf{b}}_{0} \cdot \nabla M_{j}^{(k+1)}+\frac{q_{j}}{m_{j}} k E_{\|} \nabla M_{j}^{(k-1)}
$$

where $E_{\|}=-\widehat{\mathbf{b}}_{0} \cdot \nabla \Phi$. Upon multiplication of Eq.(14) by $q_{j}$ and summation over species, we obtain (for $k=0$ )

$$
\frac{\partial \rho}{\partial t}=-\mathbf{V}_{E} \cdot \nabla \rho-\nabla_{\|} J_{\|}
$$

where $\rho=\sum_{j} q_{j} M_{j}^{(0)}$ is the charge density and $J_{\|}=\sum_{j} q_{j} M_{j}^{(1)}$ is the current density. The elliptic equation governing $\varphi$ can be calculated by taking the time derivative of the gyrokinetic Poisson equation, Eq.(7), together with Eq.(15) with the result of (in gyrokinetic units)

$$
\nabla_{\perp}^{2} \varphi=\mathbf{V}_{E} \cdot \nabla \rho+\nabla_{\|} J_{\|}
$$

Using representation (11) the gyrokinetic Poisson equation becomes

$$
\nabla_{\perp}^{2} \Phi-\left(1+\frac{1}{\tau}\right) \Phi=\int_{-\infty}^{+\infty}\left(h_{e}-h_{i}\right) d v_{\|}+Q(\Phi)
$$

where $Q(\Phi) \equiv \exp (\Phi)-\exp (-\Phi / \tau)-(1+1 / \tau) \Phi$ and $\tau=T_{i} / T_{e}$. In summary, the equations describing the splitting scheme are the equations of motions [Eqs.(4,5)], Eq.(12) for the nonadiabatic weight, and the elliptic equations $(17,16)$ for $\Phi$ and $\varphi=\partial \Phi / \partial t$, respectively. The linear dispersion relation, based on the splitting scheme, is (Appendix A)

$$
\left(1+\frac{1}{\tau}+b\right) \omega=-\omega\left[\zeta_{e} Z\left(\zeta_{e}\right)+\frac{1}{\tau} \zeta_{i} Z\left(\zeta_{i}\right)\right]+\omega_{\star}\left[\zeta_{e} R\left(\zeta_{e}\right)-\zeta_{i} R\left(\zeta_{i}\right)\right]
$$

where $R\left(\zeta_{j}\right) \equiv\left(1-\eta_{j} / 2\right) Z\left(\zeta_{j}\right)+\eta_{j} \zeta_{j}\left[1+\zeta_{j} Z\left(\zeta_{j}\right)\right], \zeta_{j} \equiv \omega /\left(\sqrt{2} k_{\|} V_{t h j}\right), b=k_{y}{ }^{2} \rho_{\mathrm{s}}{ }^{2}$ and $Z(\zeta)$ is the plasma dispersion function of Fried and Conte ${ }^{6}$. 


\section{THE MULTIGRID POISSON SOLVER}

One consequence of the splitting scheme is that the original (linear) gyrokinetic Poisson equation, Eq.(7), is transformed into a nonlinear elliptic problem, Eq.(17). It can be argued that the nonlinear term, $Q(\Phi)$, in the new form of the gyrokinetic Poisson is small [i.e.

$\mathcal{O}\left(|\Phi|^{2}\right)$ ] and that it can be neglected. However, in order to compare the $\delta f$ scheme and the splitting scheme, it is preferable to solve Eq.(17) without approximation.

In this section, we present a multigrid solver for a periodic elliptic problem. The model problem considered here is linear; the generalization of the algorithm to nonlinear elliptic equations is discussed in the next section.

\section{A. General Description}

For a configuration with period $L$, Poisson's equation is of the form

$$
L F \equiv \frac{d^{2} F}{d \theta^{2}}=S(\theta)
$$

where $\theta \equiv 2 \pi x / L$ is an angle-like coordinate with period $2 \pi$ and $S(\theta)$ is a known source term; the uniform computational grid has $N_{\theta}$ nodes. The one-dimensional problem described by Eq.(19) can be solved for $F(\theta)$ ( $\propto$ electrostatic potential) using the Fast Fourier Transform (FFT) technique. However, the nonlinear version of problem (19) is straightforward in multigrid algorithms, whereas FFT-based techniques must resort to convolutions in k space. In this paper, we choose a solution method based on multigrid techniques.

Although the advantage of using a multigrid method for the solution of Eq.(19) is not apparent (in view of the simplicity of the problem), the generalization to 2-dimensional and 3-dimensional problems in complex (e.g. toroidal) geometries is relatively easy, whereas other methods are difficult to implement, numerically inefficient or may simply not be applicable.

Consider the example of a toroidal configuration with a shaped cross-section. One can write the solution of a typical elliptic type problem in terms of Fourier-Bessel series ${ }^{7}$. In the simplest situation, the FFT technique can be used to resolve components that vary in the 
periodic coordinates (e.g. the poloidal and toroidal coordinates), whereas a one-dimensional (e.g. finite element) solver is used to treat the remaining (radial) coordinate. However the computational cost of such a scheme becomes prohibitive as the number of grid points is increased. Furthermore, as typical particle-in-cell simulations require the global solution of elliptic Poisson-type equations at each time step, it is imperative to use near-optimal global solvers, such as multigrid solvers.

An additional attractive feature of the multigrid method is that it is relatively easy to parallelize as it is amenable to domain decomposition techniques. This advantage is particularly attractive for modern, global, parallel PIC codes.

The multigrid technique ${ }^{8-10}$ is based on a set of overlapping grids. The fundamental idea behind the multigrid method is to solve modified problems using different scales (grids) in order to suppress error components of different scales. Basic iterative methods (e.g. Jacobi method, Gauss-Seidel method,...) are very efficient at damping short wavelength error components but converge poorly for the long wavelength error components.

Multigrid methods, and the closely related multilevel methods, are based on a sequence of problems of various sizes that are solved iteratively; intergrid transfer operators (prolongation and restriction operators) are used to transfer information between the solution spaces on each level. The multigrid procedure for solving elliptic partial differential equations (PDes) is stable and it uses an optimal order of time and space simultaneously. For many problems multigrid lowers the work estimate by a polylog factor; for applications with a large number of unknowns (say in the range $10^{4}-10^{6}$ ) this is a quite substantial speed up.

Problem (19) can be defined on a set of overlapping grids (or levels) as

$$
L^{(p)} F^{(p)}=S^{(p)},
$$

where $p=0,1, \cdots, Q$, and $Q$ is the total number of levels compatible with the original number of grid points $N_{\theta}$; the grid spacing on the finest grid $(p=0)$ is $\Delta \theta=2 \pi / N_{\theta}$, whereas the grid spacing on coarser grids is $\delta \theta=2^{p} \Delta \theta$ (for $p>0$ ). If $f$ denotes the exact 
solution of Eq.(19), that is $f=L^{-1} S$, and if $F$ denotes an approximation to the exact solution, then the algebraic error, $e \equiv f-F$, and the residual $r \equiv S-L F$ and related through the residual equation

$$
L e=r \quad\left(\text { or } L^{(p)} e^{(p)}=r^{(p)} .\right)
$$

If $A^{(p)}$ denotes an approximation to $L^{(p)^{-1}}$ then $F^{(p)}=A^{(p)} S^{(p)}$; in general, the approximate solution $F^{(p)}$ will depend on the initial guess $F_{0}^{(p)}$. Thus

$$
F^{(p)}=A^{(p)}\left(S^{(p)} ; F_{0}^{(p)} ; \nu_{p}\right)
$$

where $\nu_{p}$ is the number of relaxation sweeps on level $p$ and $F_{0}^{(p)}$ is the initial guess. We define the intergrid transfer operators from coarse grid to fine grid , $I(p+1 \mapsto p)$ (prolongation operator), and from fine grid to coarse grid, $I(p \mapsto p+1)$ (restriction operator); we have the relations

$$
F^{(p)}=I(p+1 \mapsto p) F^{(p+1)}
$$

and

$$
F^{(p+1)}=I(p \mapsto p+1) F^{(p)}
$$

where $p=0,1, \cdots, Q-1$. Note that intergrid transfer operators are local and therefore approximate. As a consequence the convolutions of intergrid transfer operators

$$
I(p \mapsto p+1) I(p+1 \mapsto p) \text { and } I(p+1 \mapsto p) I(p \mapsto p+1)
$$

are, in general, not equal to the identity operator $\widehat{I}$; the notable exception is, of course, the linear profile. Here we consider a simple multigrid $V$ cycle algorithm; in the $V$ cycle, one proceeds from the finest grid $(p=0)$ to the coarsest grid $(p=Q)$, and then back to the finest grid. The $V$ cycle can be cast in the following algorithmic form of

\section{MultiGrid V cycle}




$$
\begin{aligned}
F^{(p)} & =A^{(p)}\left(S^{(p)} ; F_{0}^{(p)} ; \nu_{p}\right) \\
r^{(p)} & =S^{(p)}-L^{(p)} F^{(p)} ; p=0,1, \cdots, Q-1 \\
S^{(p+1)} & =I(p \mapsto p+1) r^{(p)} \\
\ldots \ldots & \cdot \cdots \ldots \ldots \ldots \ldots \ldots \ldots \ldots \ldots \\
F_{0}^{(p)} & \Longleftarrow F^{(p)}+I(p+1 \mapsto p) F^{(p+1)} \\
F^{(p)} & =A^{(p)}\left(S^{(p)} ; F_{0}^{(p)} ; \nu_{p}\right)
\end{aligned}
$$

The dotted line indicates the bottom of the $V$ cycle. The numerical solution of a typical elliptic problem (19) is presented in the next section.

\section{B. Numerical Example}

In this section, we present some numerical experiments based on the algorithm presented in the previous section. The source term in Eq.(19) is taken to be of the form

$$
S(\theta)=-\sum_{q=1}^{M} q^{2}\left[\widehat{\alpha}_{q} \cos (q \theta)+\widehat{\beta}_{q} \sin (q \theta)\right],
$$

which corresponds to the exact solution

$$
f(\theta)=\sum_{q=1}^{M} \widehat{\alpha}_{q} \cos (q \theta)+\sum_{q=1}^{M} \widehat{\beta}_{q} \sin (q \theta),
$$

where $M$ is the total number of modes; such exact solution allows us to 'tune' specific modes and study the performance of the multigrid solver for short-, intermediate- and longwavelength modes. All the numerical results presented in this section are for $M=12$ modes with amplitudes $\widehat{\alpha}_{q}=\widehat{\beta}_{q}=1$, for $q=1,2, \cdots, M$. The initial profile (on the finest grid) is chosen as

$$
F_{0}(\theta)=\pi^{2} \sin (\theta / 2)
$$

Figure 1 shows the $L^{2}$ norm of the residual as a function of the number of the $V$ cycles for a grid with $N_{\theta}=256$ nodes. The number of relaxations per level is kept fixed at $\nu=4$; 
the basic solver on each level is the damped Jacobi method with damping parameter $\omega=$ 0.6. The restriction operator used is the full weighting operator, whereas the prolongation operator is the linear interpolation operator. We note the sharp decrease in the $L^{2}$ norm of the residual after a single $\mathrm{V}$ cycle. The discretization error is reached after $9 \mathrm{~V}$ cycles. In order to illustrate the efficiency of the multigrid algorithm, the same problem was solved (with the same parameters and the same initial conditions) using the damped Jacobi method on the finest grid, that is no coarser grids were used during the numerical solution. As shown in Figure 2, the damped Jacobi solver performs well in the first iterations, and its efficiency then decreases dramatically; this is the signature that the long wavelength modes are not properly resolved. To put the difference in efficiency in perspective, a total of 6846 iterations were required for the single-grid Jacobi solver to reach the same residual norm as that obtained by a set of 10 multigrid $V$ cycles! This dramatic difference between the 2 solvers becomes more acute as the number of grid points increases.

Figure 3 shows the initial and exact profiles, as well as the approximate profile, obtained after one $V$ cycle. The multigrid solver 'picks up' the right modes after a single iteration, but the amplitudes are not those of the exact solution. After $3 V$ cycles, the approximate amplitudes are in much better agreement with the exact amplitudes (Figure 4). After $7 \mathrm{~V}$ cycles, the approximate solution is virtually indistinguishable for the exact solution (Figure $5)$.

\section{NUMERICAL EXPERIMENTS}

The multigrid solver described in the previous section is now used to compare the standard $\delta f$ scheme and the splitting scheme for the case of electrostatic drift waves. All simulations were carried out with $m_{i} / m_{e}=1836$.

We first discuss some linear simulations. The parameters for Figures $(6,7)$ are for a set of $N_{i}=6765$ ion markers, $N_{e}=6765$, on a 64 -grid system of length $L=8$; the time step is $\Delta t=1$. The magnetic field tilt is $\theta=0.01$, and the electron and ion temperature-gradient 
parameters are $\eta_{e}=\eta_{i}=0$. Markers were initially loaded using the quiet start technique based on Fibonacci numbers. ${ }^{11}$. The driving parameter, $\kappa=\rho_{s} / L_{n}$, has been varied between 0.05 and 0.13 .

For the splitting scheme, the gyrokinetic Poisson equation, Eq.(17), has been solved using the multigrid solver described in the previous section. Newton's method is used to treat the term $Q(\Phi)$ in Eq.(17); typically 2 to 3 Newton iterations are sufficient for convergence. For all the simulations presented in this paper, the multigrid relaxations have been carried out with a set of $6 \mathrm{~V}$ cycles, with $\nu=4$ relaxations on each grid level.

The mode frequency, $\omega_{r}$, obtained from the $\Phi$ power spectrum, for the $\delta f$ and splitting schemes is shown in Figure 6. The plain line represents the numerical solution (based on Muller's algorithm ${ }^{12}$ in the complex $\omega_{r}-\gamma$ plane) of the exact linear dispersion relation (18). Both schemes predict mode frequency in reasonable agreement with the exact solution. However, the comparison of the linear growth rates is more meaningful. As shown in Figure 7, the splitting scheme yields a linear growth in excellent agreement with the theory; the standard $\delta f$ scheme, however, is quite inaccurate even when the drive is strong. Increasing the number of markers per cell should improve the agreement between the $\delta f$ and theoretical results. This is shown in Figure 8 for the case of $N_{i}=N_{e}=10946$ markers (all other parameters are the same as in Figure 7). However inaccurate linear growth rates are still observed at low drive. Increasing the grid resolution while keeping the number of markers per cell constant does not alter the results of Figure 7 significantly.

The favorable numerical properties of the splitting scheme can be best understood from the power spectrum of the electrostatic potential. Figure 9 shows the power spectrum based on the $\delta f$ scheme for a linear run with 500 time steps (linear run) (in Figs. $(9,10)$, $[\Phi]$ stands for $|\Phi|^{2}$ ). The parameters are $L=8$ (system size), $\Delta x=1 / 8$ (grid spacing), $N_{e}=N_{i}=10946, \kappa=0.05, \eta_{e}=\eta_{i}=0$ and $\theta=0.01$. We note the presence of a 'secondary mode' (near $\omega=0$ ) and the slow decay of the high-frequency tail. Figure 10 shows the power spectrum using the splitting scheme based on 500 time steps; all parameters are the same as those of Figure 9; the initial configuration in phase space in Figure 9 and Figure 
10 are identical. The spectrum has no 'secondary mode' (compare with Figure 9). Most importantly, $P(\omega)$ decays with $\omega$ much faster compared with the $\delta f$ run. The presence of a single, well-defined maxima in $P(\omega)$ and the rapid decay with $\omega$ is a signature that the nonadiabatic electrons have not 'contaminated' the plasma response, as was the case in the $\delta f$ scheme. Based on Figures 9 and 10, one can intuitively expect a better agreement with theory for the linear growth rate using the splitting scheme, as was oberved in Figure 7.

Figure 11 shows the real and imaginary parts of the electrostatic potential for a nonlinear run. The parameters are $N_{e}=N_{i}=10946$ and $\kappa=0.15$. Other parameters are the same as in Figure 10. We note that this clean result has been obtained for a relatively small number of markers.

The nonlinear saturation of the most unstable modes $(n \pm 1)$ is due to the parallel nonlinearity; in this simplified one-dimensional model, the saturation due to the $\mathbf{E} \times \mathbf{B}$ advection is absent. In a more realistic situation (e.g. toroidal plasmas), both mechanisms can contribute to saturate the drift waves. Based on a perturbative, three-wave coupling theory (two fastest growing modes and the nonlinear $n=0$ response due to mode coupling), Parker and Lee have estimated that the saturation level of $\Phi(n=1)$ to be ${ }^{13}$

$$
|\Phi|_{\mathrm{sat}}=5.48\left(\frac{\gamma_{\ell}}{k_{\|} V_{\text {the }}}\right)^{2}
$$

where $\gamma_{\ell}$ is the linear growth rate and $k_{\|}=\theta k_{\perp}$ in this one-dimensional model. Figure 12 shows the measured saturation level for the $n=1$ mode as a function of the drive, $\kappa$, for a simulation with $N_{i}=N_{e}=6765$ markers. Since the linear growth rate varies linearly with the density gradient parameter, we expect $|\Phi|_{\text {sat }} \propto \kappa^{2}$. The plain line in Figure 12 shows the theoretical value [Eq.(29)] whereas the squares and the triangles correspond to the measured values for the splitting scheme and the $\delta f$ scheme, respectively. Taking into account that these simulations have been carried out with a small number of markers $\left(N_{i}=N_{e}=6765\right)$, the agreement with theory is quite satisfactory. We note, however, a departure from the theoretical curve for strong drive; this trend is more pronouced for the $\delta f$ scheme than for the splitting scheme. 


\section{CONCLUDING REMARKS}

We have presented multigrid particle-in-cell simulations of electrostatic drift waves with kinetic electrons. The impact of electron dynamics on the anomalous transport ${ }^{3,4}$ in toroidal devices is expected to be quite substantial; this impact could be more pronounced in stellarator plasmas ${ }^{14}$ where toroidally-trapped and helically-trapped electron population coexist.

For illustrative purposes, we have compared the standard $\delta f$ scheme with the splitting scheme, which only treats the nonadiabatic electron response dynamically, for a onedimensional case. For identical parameters (same number of markers, same configuration in phase space at $t=0$,etc...), it has been shown that the splitting scheme yields better results

for the linear growth rates and for the mode frequency. Furthermore, the power spectrum of the electrostatic potential shows that the splitting scheme does not suffer from the contamination of the nonadiabatic electrons. Finally, the saturation of the fastest growing modes due to the parallel nonlinearity has been shown to be in good agreement with the three-wave coupling theory ${ }^{13}$ even for a small number of markers.

This paper represents the first example of what we term the "multigrid particle-in-cell concept' applied to plasma microturbulence. Although a relatively simple (but nonlinear) problem was presented here, the generalization to complex geometry (e.g. nonaxisymmetric, toroidal plasmas) is far easier using the multigrid approach compared to other methods that usually rely on various (and sometimes dubious) approximations and/or are plagued with numerical inefficiency.

\section{ACKNOWLEDGMENTS}

This research was supported by Contract No DE-AC02-76CH03073 and the Scientific Discovery through Advanced Computing (SciDAC) initiative (U.S. Department of Energy). 


\section{APPENDIX A: LINEAR PROPERTIES OF ELECTROSTATIC DRIFT WAVES}

In this Appendix, we derive the dispersion relation for electrostatic drift waves based on the splitting scheme. The distribution for species $j$ is written as $F_{j}=\exp \left(-q_{j} \Phi / T_{j}\right) F_{M j}+h_{j}$ where the nonadiabatic part, $h_{j}$, is governed by

$$
\frac{d h_{j}}{d t}=\exp \left(-\frac{q_{j} \Phi}{T_{j}}\right)\left[\mathbf{V}_{E} \cdot \boldsymbol{\kappa}_{j}+\frac{q_{j}}{T_{j}} \frac{\partial \Phi}{\partial t}\right] F_{M j}
$$

where $\boldsymbol{\kappa}_{j}=\boldsymbol{\kappa} g_{j}\left(\bar{v}_{\|}\right) ; g_{j}\left(\bar{v}_{\|}\right)=1-\eta_{j}\left(1-\bar{v}_{\|}^{2}\right) / 2$; and $\bar{v}_{\|}=v_{\|} / V_{t h j}$. Assuming perturbations of the form $\exp (i \mathbf{k} \cdot \mathbf{r}-i \omega t)$ and linearizing, we get

$$
h_{j}=\left[\omega_{\star} g_{j}\left(\bar{v}_{\|}\right)+\sigma_{j} \omega\right] \frac{F_{M j}}{\omega-k_{\|} v_{\|}} \widetilde{\Phi}
$$

where $\omega_{\star}=\left(k_{y} \rho_{\mathrm{s}}\right) c_{s} / L_{n}$ is the drift frequency, $\sigma_{j}=Z_{j} T_{e} / T_{j}$ and $\widetilde{\Phi}=e \Phi / T_{e}$. Multiplying Eq.(A2) by $v_{\|}^{k}$ and integrating over velocity space

$$
\int_{-\infty}^{+\infty} h_{j} v_{\|}^{k} d v_{\|}=-n_{0} \frac{\left(\sqrt{2} V_{t h j}\right)^{k}}{\omega_{\| j}}\left\{\left[\sigma_{j} \omega+\omega_{\star}\left(1-\eta_{j} / 2\right)\right] Z^{(k)}\left(\zeta_{j}\right)+\omega_{\star} \eta_{j} Z^{(k+2)}\left(\zeta_{j}\right)\right\} \widetilde{\Phi}
$$

where $\omega_{\| j} \equiv \sqrt{2} k_{\|} V_{t h j}, \zeta_{j} \equiv \omega / \omega_{\| j}$; we have defined

$$
Z^{(k)}(\zeta) \equiv \frac{1}{\sqrt{\pi}} \int_{-\infty}^{+\infty} \frac{x^{k}}{x-\zeta} \exp \left(-x^{2}\right) d x
$$

for nonnegative integer $k ; Z^{(0)}(\zeta) \equiv Z(\zeta)$ is the usual plasma dispersion function of Fried and Conte ${ }^{6}$. The particle density for species $j$ is

$$
\begin{aligned}
n_{j} & =n_{0} \exp \left(-\frac{q_{j}}{T_{j}} \Phi\right)+\int_{-\infty}^{+\infty} h_{j} d v_{\|}, \\
& =n_{0}\left(1-\sigma_{j} \widetilde{\Phi}\right)+\int_{-\infty}^{+\infty} h_{j} d v_{\|} .
\end{aligned}
$$

Substituting Eq.(A5) in the gyrokinetic Poisson equation, $k_{y}{ }^{2} \rho_{\mathrm{s}}{ }^{2} \widetilde{\Phi}=\left(n_{i}-n_{e}\right) / n_{0}$, and using Eq.(A3), we obtain the dispersion relation for electrostatic drift waves

$$
\left(1+\frac{1}{\tau}+b\right) \omega=-\omega\left[\zeta_{e} Z\left(\zeta_{e}\right)+\frac{1}{\tau} \zeta_{i} Z\left(\zeta_{i}\right)\right]+\omega_{\star}\left[\zeta_{e} R\left(\zeta_{e}\right)-\zeta_{i} R\left(\zeta_{i}\right)\right]
$$

where $R\left(\zeta_{j}\right) \equiv\left(1-\eta_{j} / 2\right) Z\left(\zeta_{j}\right)+\eta_{j} \zeta_{j}\left[1+\zeta_{j} Z\left(\zeta_{j}\right)\right], \tau=T_{i} / T_{e}$ and $b=k_{y}{ }^{2} \rho_{\mathrm{s}}{ }^{2}$. In the cold ions, warm electrons limit 


$$
\frac{\omega}{k_{\|} V_{t h i}} \gg 1 \gg \frac{\omega}{k_{\|} V_{t h e}},
$$

Eq.(A6) assumes the simplified form of

$$
(1+b) \omega=\omega_{\star}+i \sqrt{\pi} \zeta_{e}\left[\omega_{\star}\left(1-\eta_{e} / 2\right)-\omega\right],
$$

which can be solved for the mode frequency

$$
\omega_{\mathrm{r}}=\frac{\omega_{\star}}{1+b}
$$

and the linear growth rate

$$
\begin{aligned}
\gamma & =\sqrt{\frac{\pi}{2}} \frac{\omega_{\mathrm{r}}}{k_{\|} V_{t h e}} \frac{\omega_{\star}\left(1-\eta_{e} / 2\right)-\omega_{\mathrm{r}}}{1+b} \\
& =\sqrt{\frac{\pi}{2} \frac{m_{e}}{m_{i}}} \frac{\omega_{\star}^{2}}{k_{\|} c_{s}} \frac{(1+b)\left(1-\eta_{e} / 2\right)-1}{(1+b)^{3}} .
\end{aligned}
$$




\section{REFERENCES}

${ }^{1}$ P. C. Liewer, Nucl. Fusion 25, 543 (1985).

${ }^{2}$ F. Wagner and U. Stroth, Plasma Phys. Contr. Fusion, 35, 1321 (1993).

${ }^{3}$ W. Horton, Handbook of Plasma Physics (Vol.2) (Elsevier Science, Amsterdam, 1984).

${ }^{4}$ W. Horton, Rev. Mod. Phys. 71, 735 (1999).

${ }^{5}$ R.E. Denton and M. Kotschenreuther, J. Comp. Phys. 119, 283 (1995).

${ }^{6}$ B.D. Fried and S.D. Conte, Plasma Dispersion Function (Academic Press, New York, 1961).

${ }^{7}$ J.L.V. Lewandowski, Phys. of Plasmas, 7(2), 588 (2000).

${ }^{8}$ P. Wesseling, An Introduction to Multigrid Methods (Wiley, Chichester, 1992).

${ }^{9}$ F. De la Vallee Poussin, SIAM J. of Num. Analysis, 5(2), 340 (1968).

${ }^{10}$ A. Settari and K. Aziz, SIAM J. of Num. Analysis, 10(3), 506 (1973).

11 J. Denavit and J.M. Walsh, Comments Plasma Phys. Cont. Fusion, 6, 209 (1981).

${ }^{12}$ W.S Press, S.A. Teukolsky, W.T. Vetterling and B.P. Flannery, Numerical Recipes in Fortran (Cambridge University Press, New York, 1992).

${ }^{13}$ S. Parker and W.W. Lee, Phys. Fluids, B5(1), 77 (1993).

${ }^{14}$ J.L.V. Lewandowski, Phys. of Plasmas, 7(8), 3360 (2000). 
Figure $1 L^{2}$ norm of the residual as a function of the number of $V$ cycles (iterations) for a grid with $N_{\theta}=256$ grid points. The number of relaxation sweeps on each level is $\nu=4$ and the Jacobi damping parameter is $\omega=0.6$. The initial profile is given by Eq.(28).

Figure $2 L^{2}$ norm of the residual as a function of the number of Jacobi solves on the finest grid. Other parameters are the same as in Figure 1. A total of 6846 iterations are required to reach the same residual norm as that obtained for a set of $10 \mathrm{~V}$ cycles $\left(\|r\|_{10}^{2}=0.066\right)$

Figure 3 Exact (plain line), initial (dashed line) and approximate profile (dotted line) as a function of the periodic coordinate $\theta$. The approximate profile shown is that obtained after one $V$ cycle (other parameters are $\omega=0.6, \nu=4$ and $N_{\theta}=256$ ).

Figure 4 Same as Figure 3 but the approximate profile shown is that obtained after $3 \mathrm{~V}$ cycles.

Figure 5 Same as Figure 3 but the approximate profile shown is that obtained after $7 \mathrm{~V}$ cycles.

Figure 6 Mode frequency for the standard $\delta f$ scheme (triangles) and for the splitting scheme (squares) as a function of $\kappa=\rho_{\mathrm{s}} / L_{n}$. The plain line is the numerical solution of the linear dispersion relation. The parameters are: $N_{e}=N_{i}=6765$, on a grid of length $L=8$ with 64 grid points; $\eta_{e}=\eta_{i}=0$ and $\theta=0.01$. Only the $n=1$ mode $\left(k_{\perp} \rho_{s} \simeq 0.78\right)$ is retained in the simulation. The initial configuration in phase space for the splitting scheme run and the $\delta f$ run are identical.

Figure 7 Linear growth rate for the standard $\delta f$ scheme (triangles) and for the splitting scheme (squares) as a function of $\kappa=\rho_{\mathrm{s}} / L_{n}$. The plain line is the numerical solution of the linear dispersion relation. The parameters are: $N_{e}=N_{i}=6765$, on a grid of length $L=8$ with 64 grid points; $\eta_{e}=\eta_{i}=0$ and $\theta=0.01$. Only the $n=1$ mode 
$\left(k_{\perp} \rho_{s} \simeq 0.78\right)$ is retained in the simulation. The initial configuration in phase space for the splitting scheme run and the $\delta f$ run are identical.

Figure 8 Same as Figure 7 but for $N_{i}=N_{e}=10946$ markers. The initial configuration in phase space for the splitting scheme run and the $\delta f$ run are identical.

Figure 9 Power spectrum of the electrostatic potential for the standard $\delta f$ scheme based on 500 time steps (linear run). The parameters are $L=8$, number of grid points $N_{g}=64, N_{e}=N_{i}=10946, \kappa=0.05, \eta_{e}=\eta_{i}=0$ and $\theta=0.01$.

Figure 10 Power spectrum of the electrostatic potential for the splitting scheme based on 500 time steps (linear run). The parameters and the initial configuration in phase space are identical to those of Figure 8.

Figure 11 Time history of the real (plain line) and imaginary (dotted line) parts of the $n=1$ mode based on the splitting scheme (nonlinear run).

Figure 12 Saturation level of the Parker-Lee three-wave mode coupling theory ${ }^{13}$ (plain line) compared with the measured values for the splitting scheme (squares) and the $\delta f$ scheme (triangles) for a set of $N_{e}=N_{i}=6765$ as a function of the drive, $\kappa$. 


\section{FIG.1 Lewandowski}

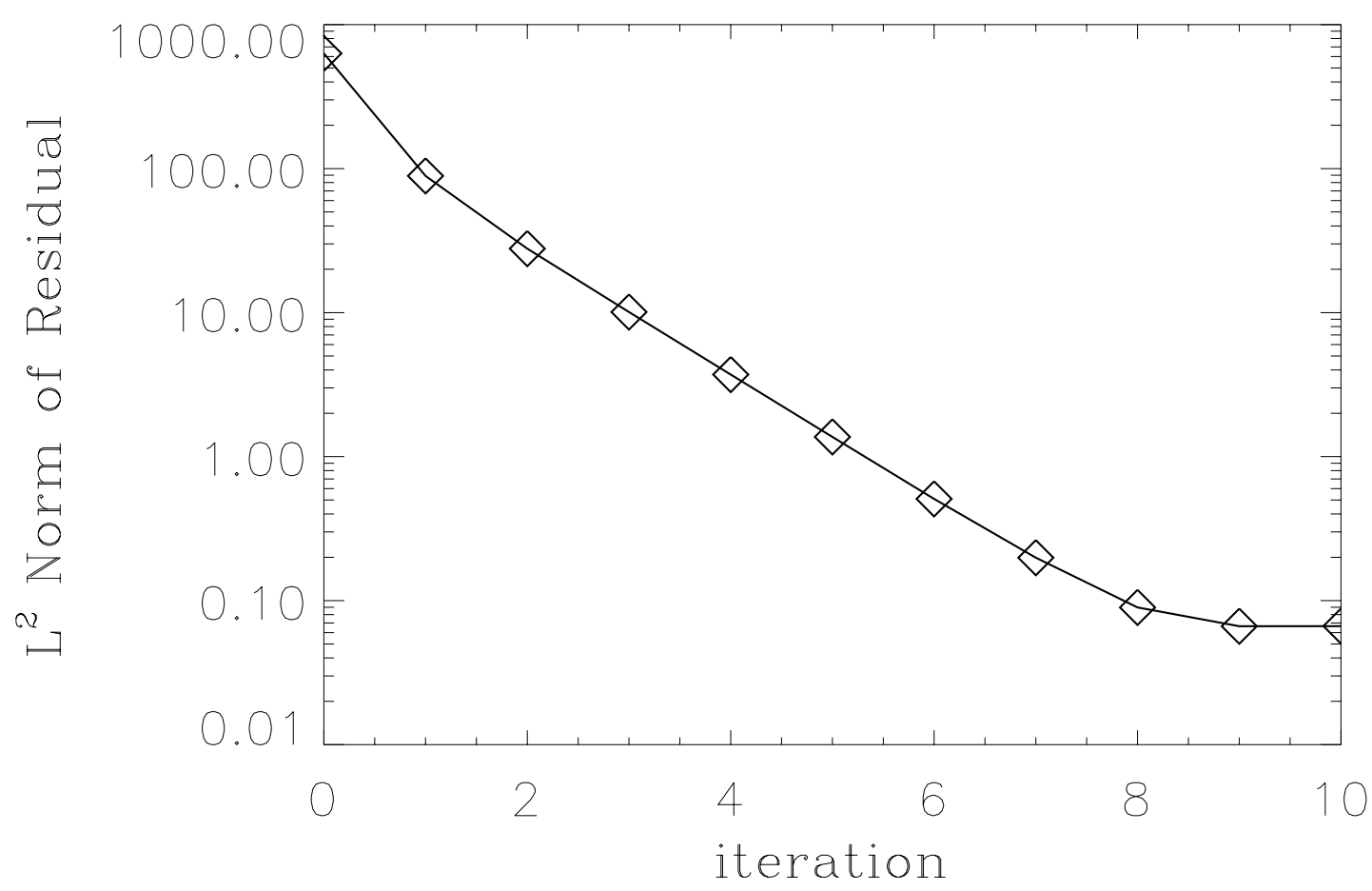




\section{FIG.2 Lewandowski}

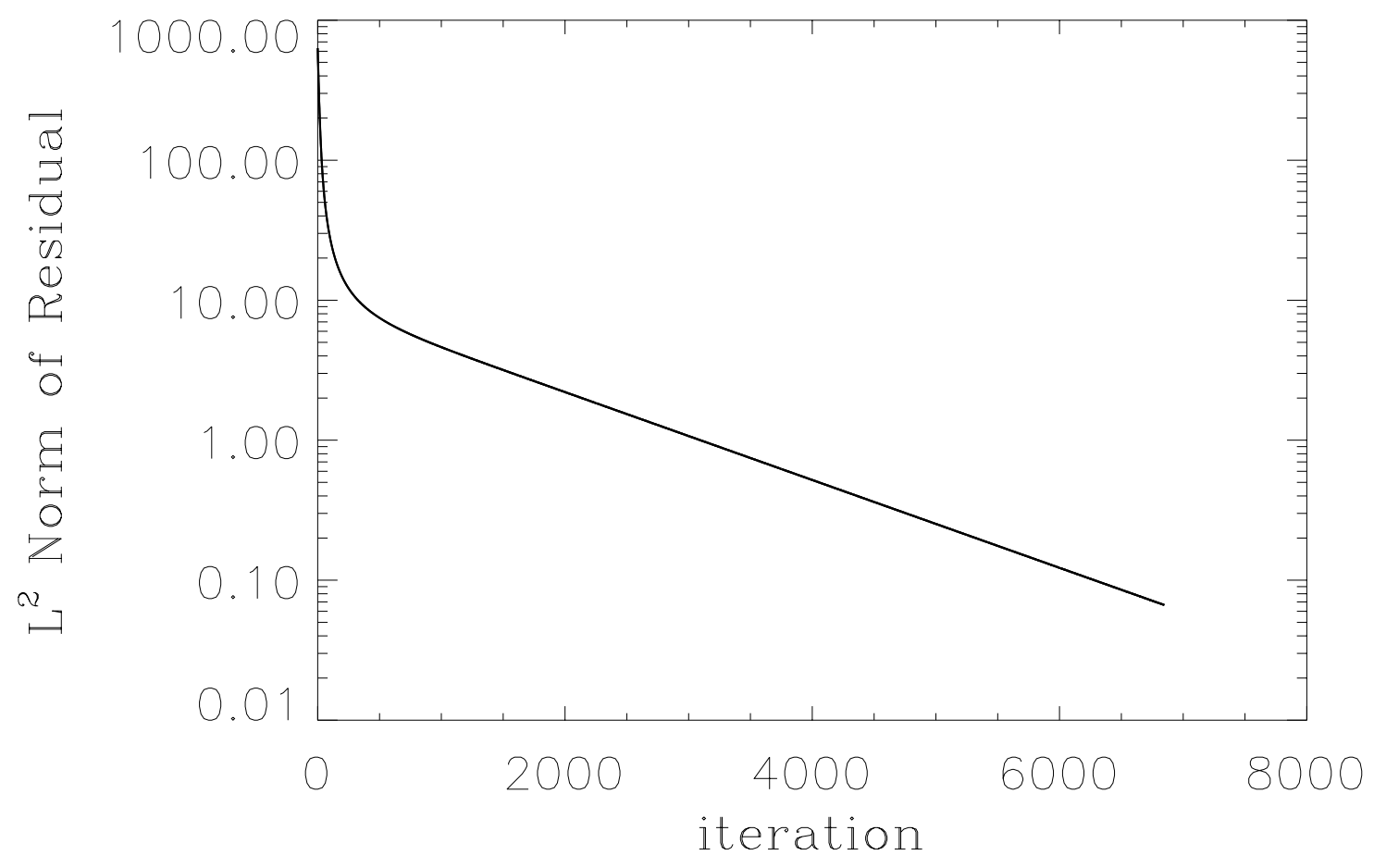




\section{FIG.3 Lewandowski}

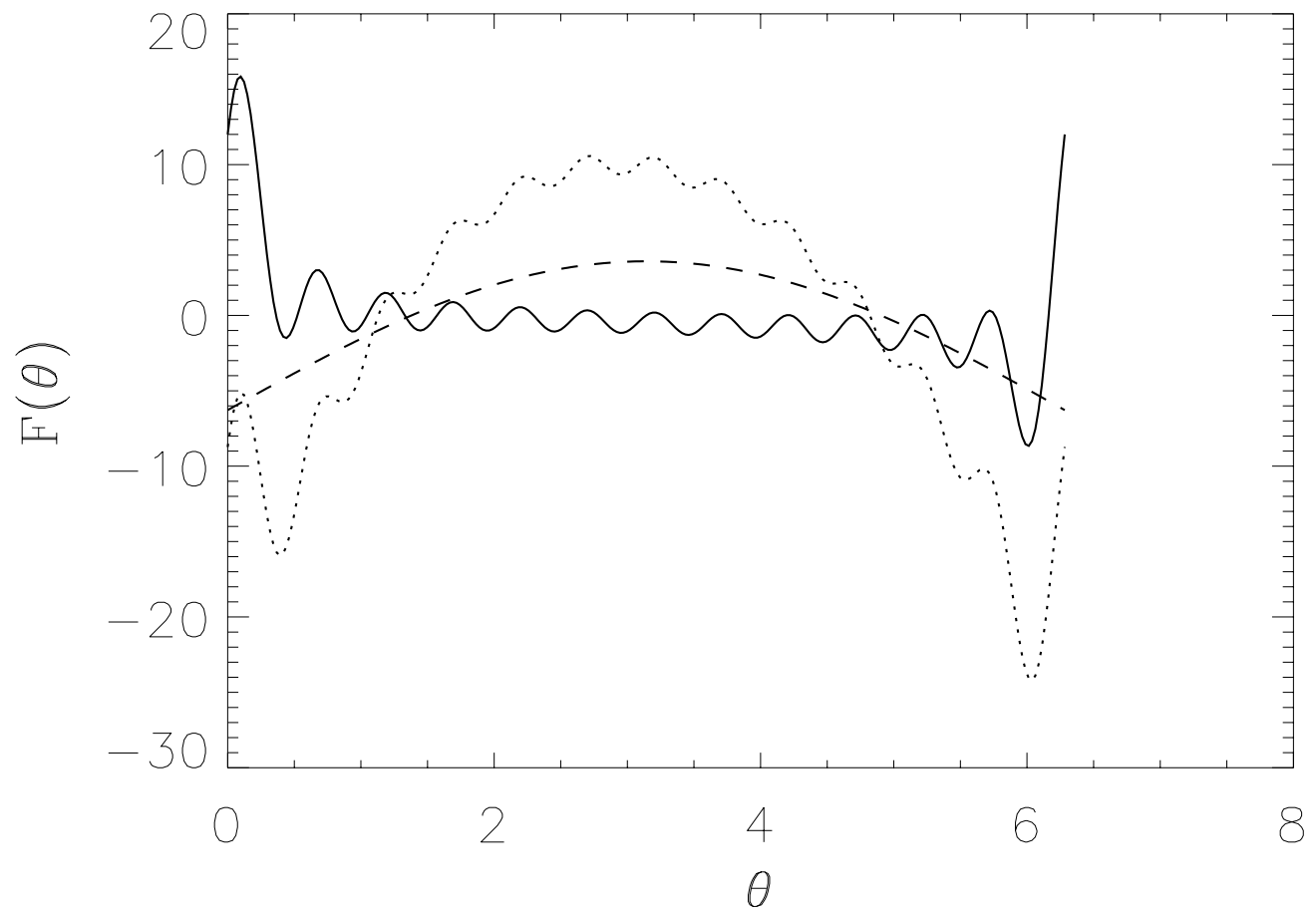




\section{FIG.4 Lewandowski}

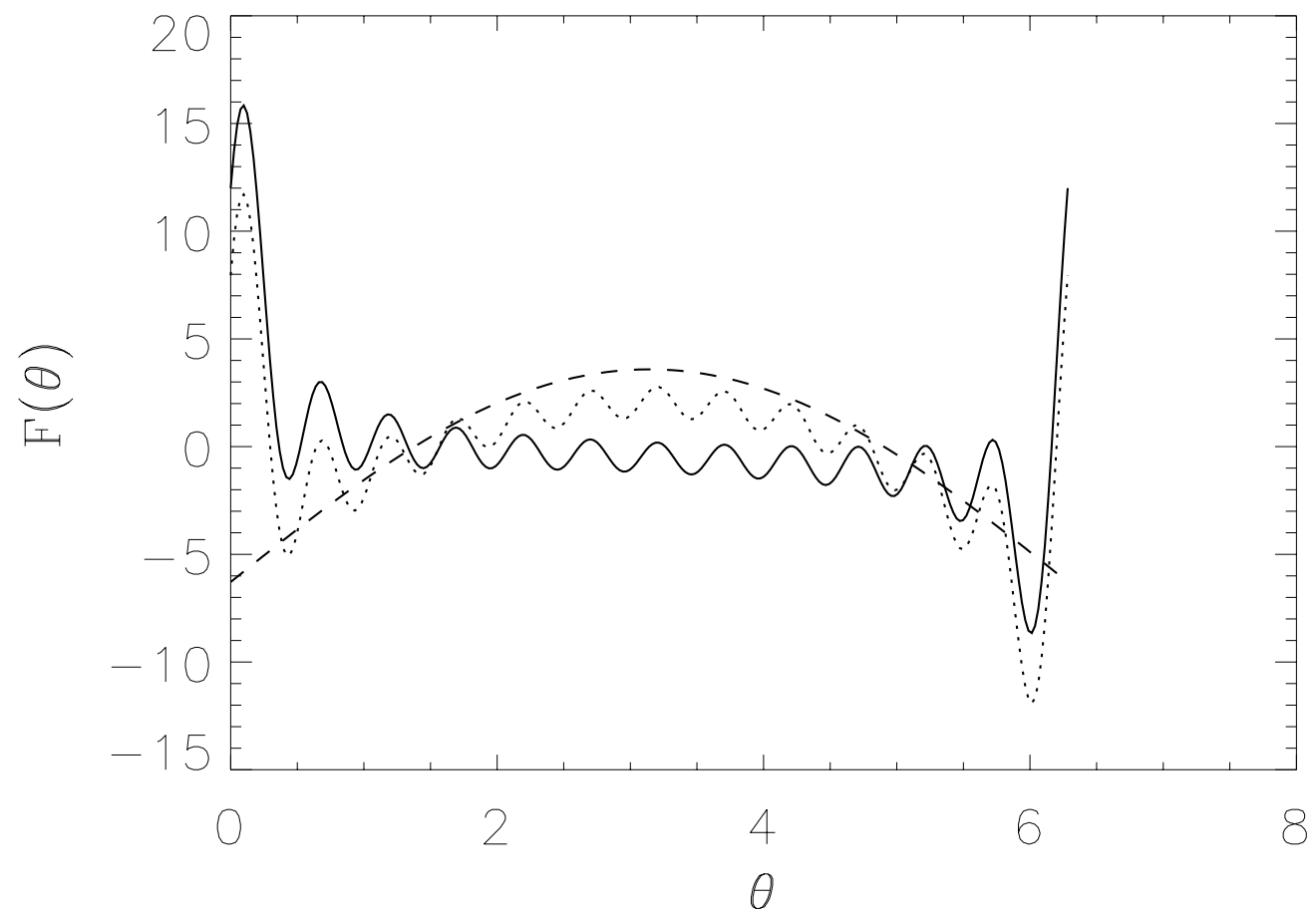


FIG.5 Lewandowski

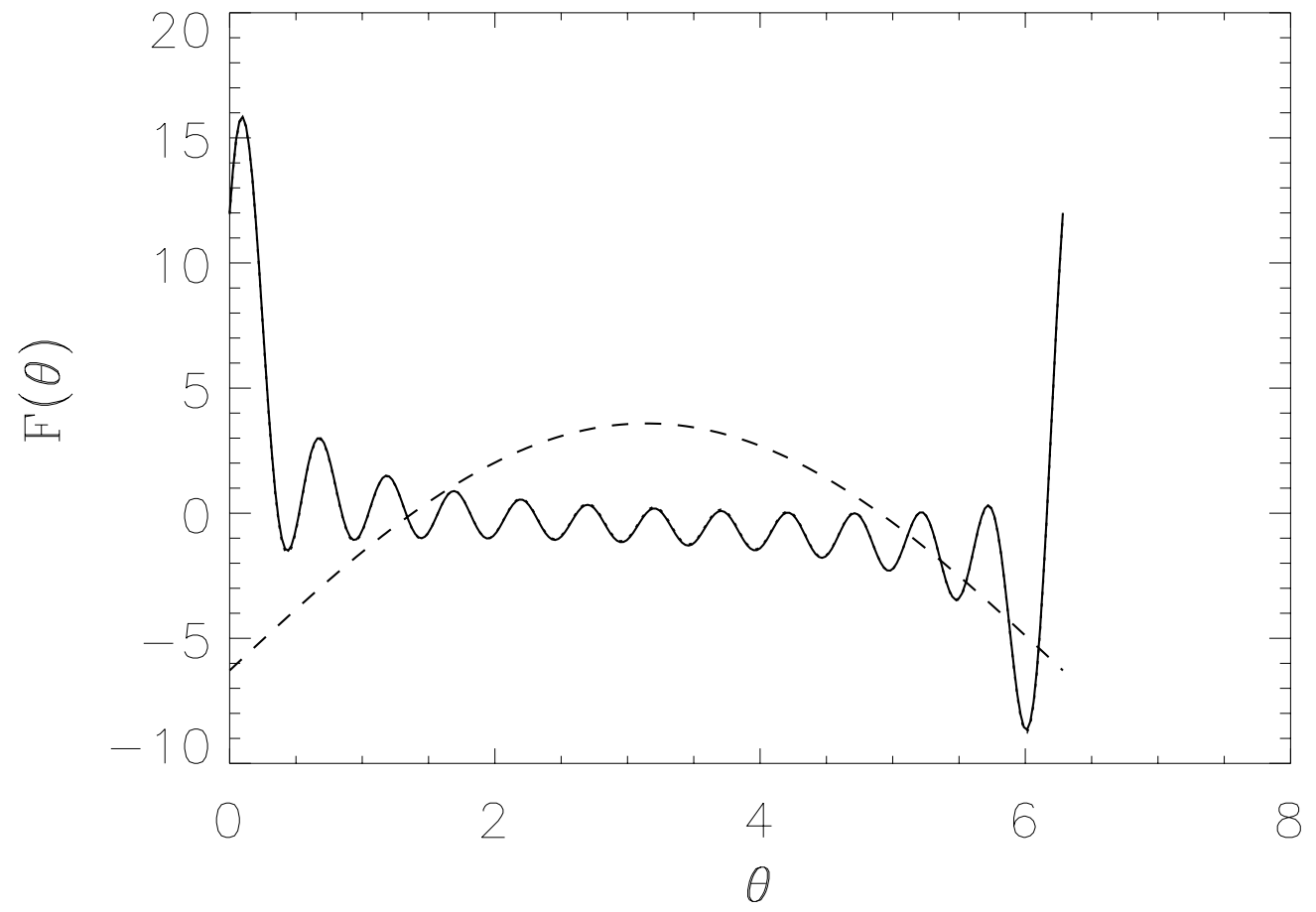




\section{FIG.6 Lewandowski}

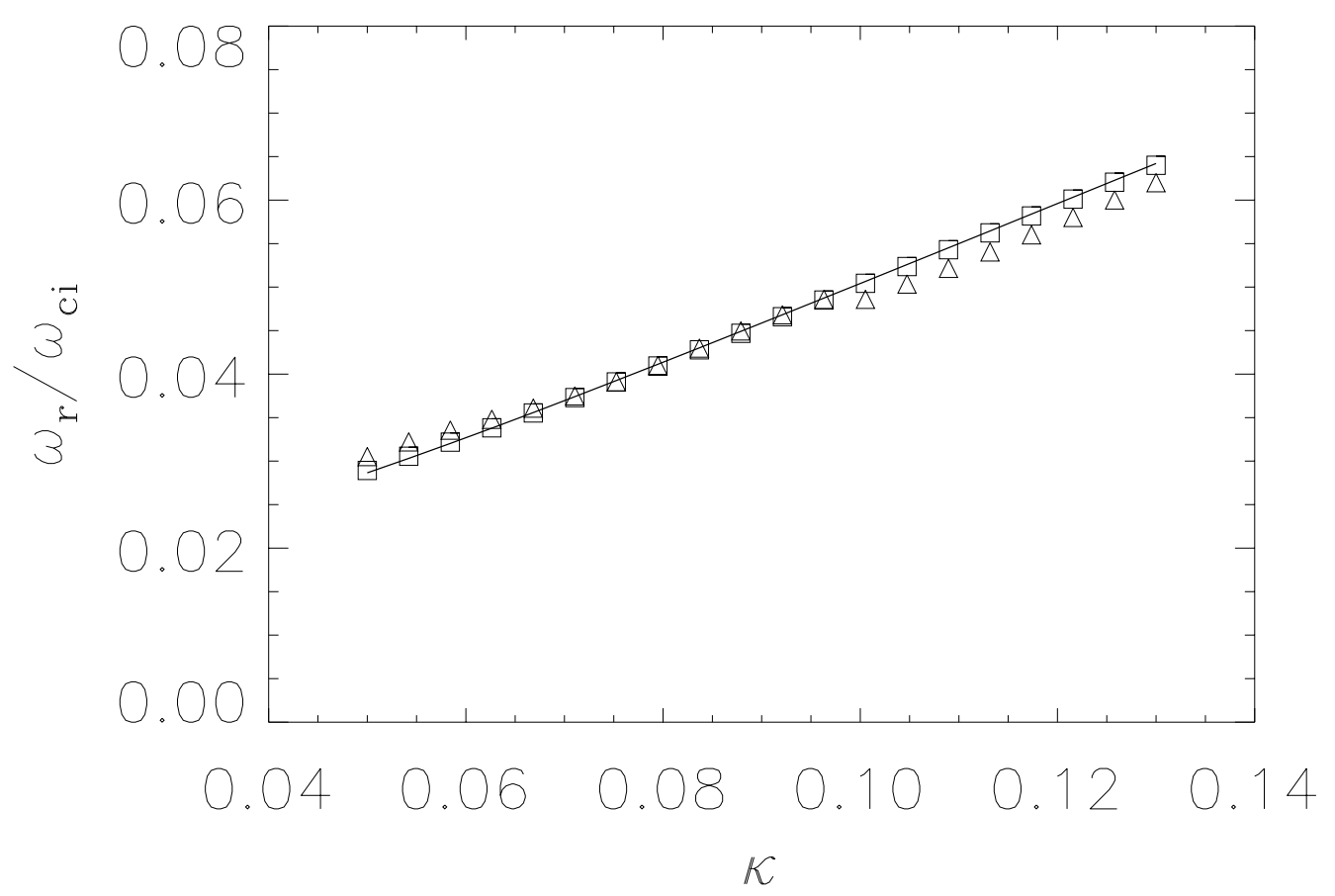




\section{FIG.7 Lewandowski}

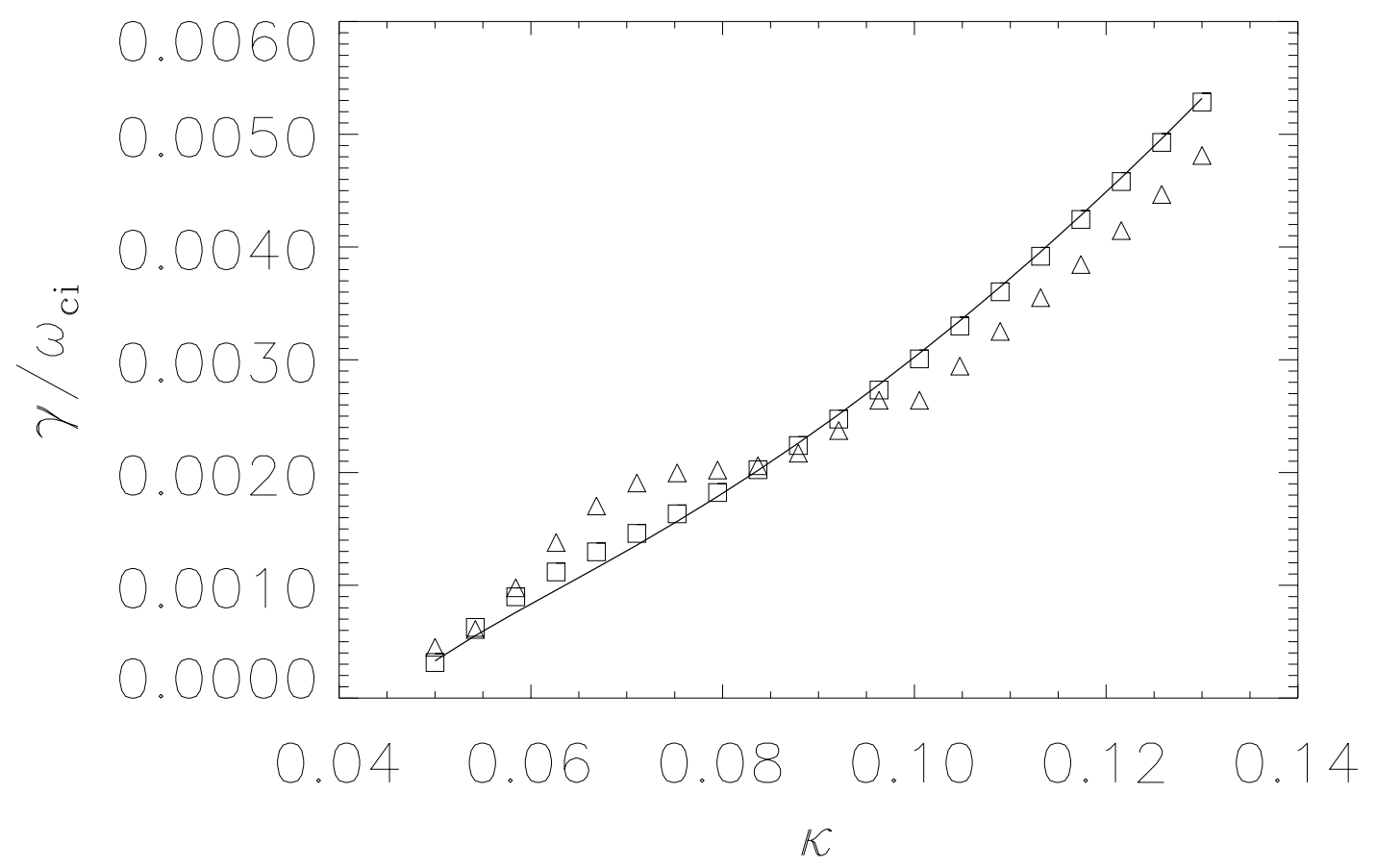




\section{FIG.8 Lewandowski}

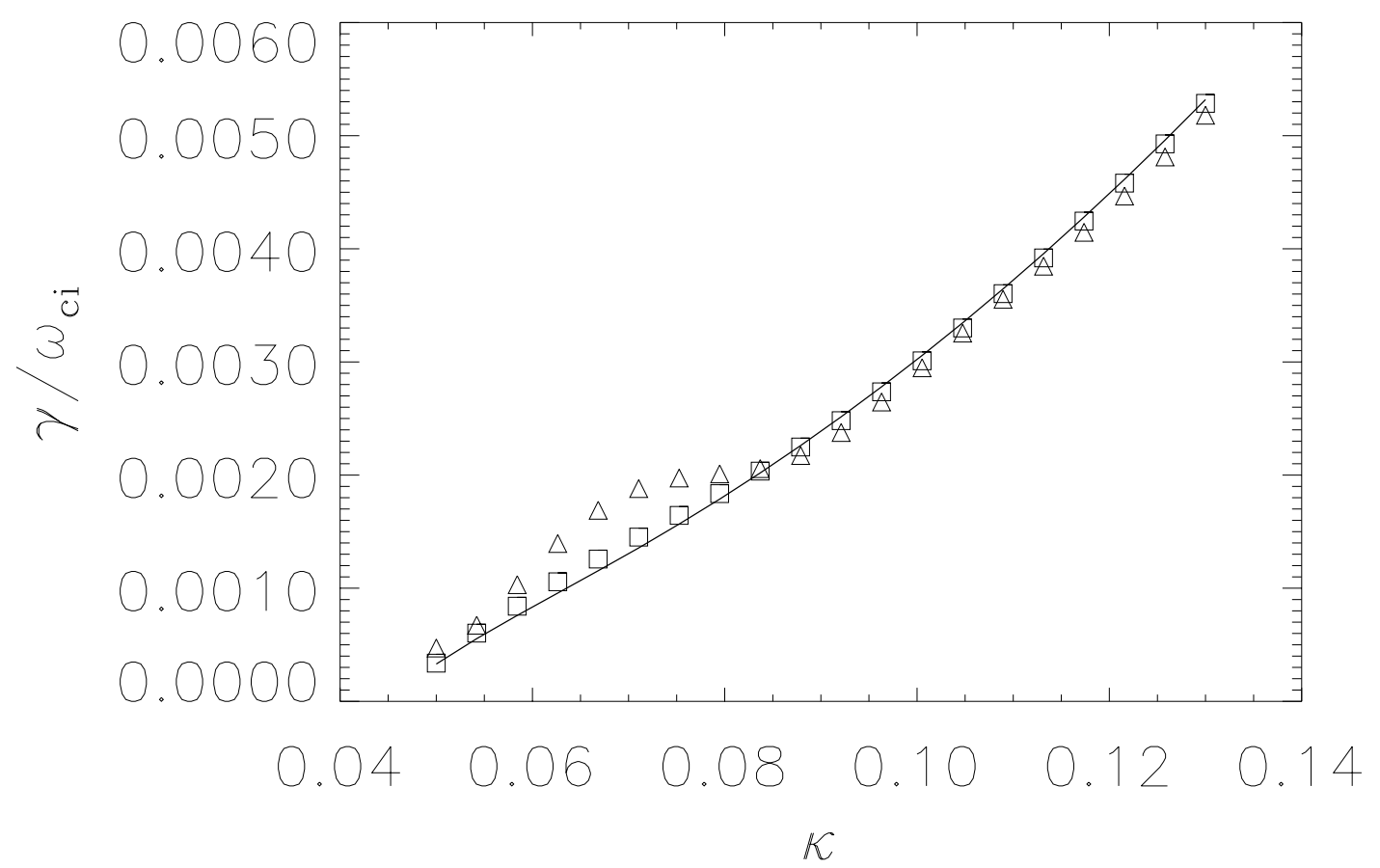




\section{FIG.9 Lewandowski}

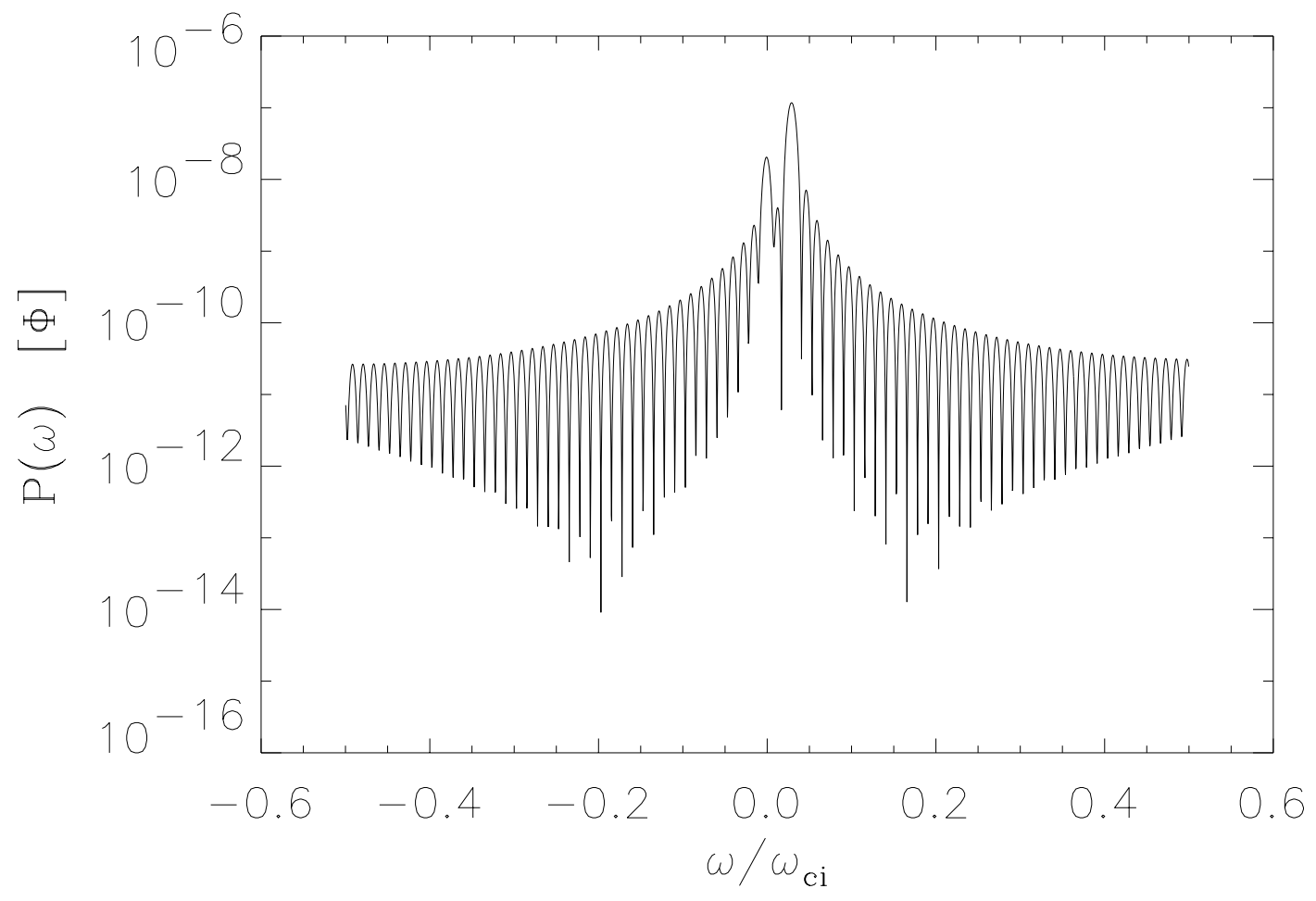




\section{FIG.10 Lewandowski}

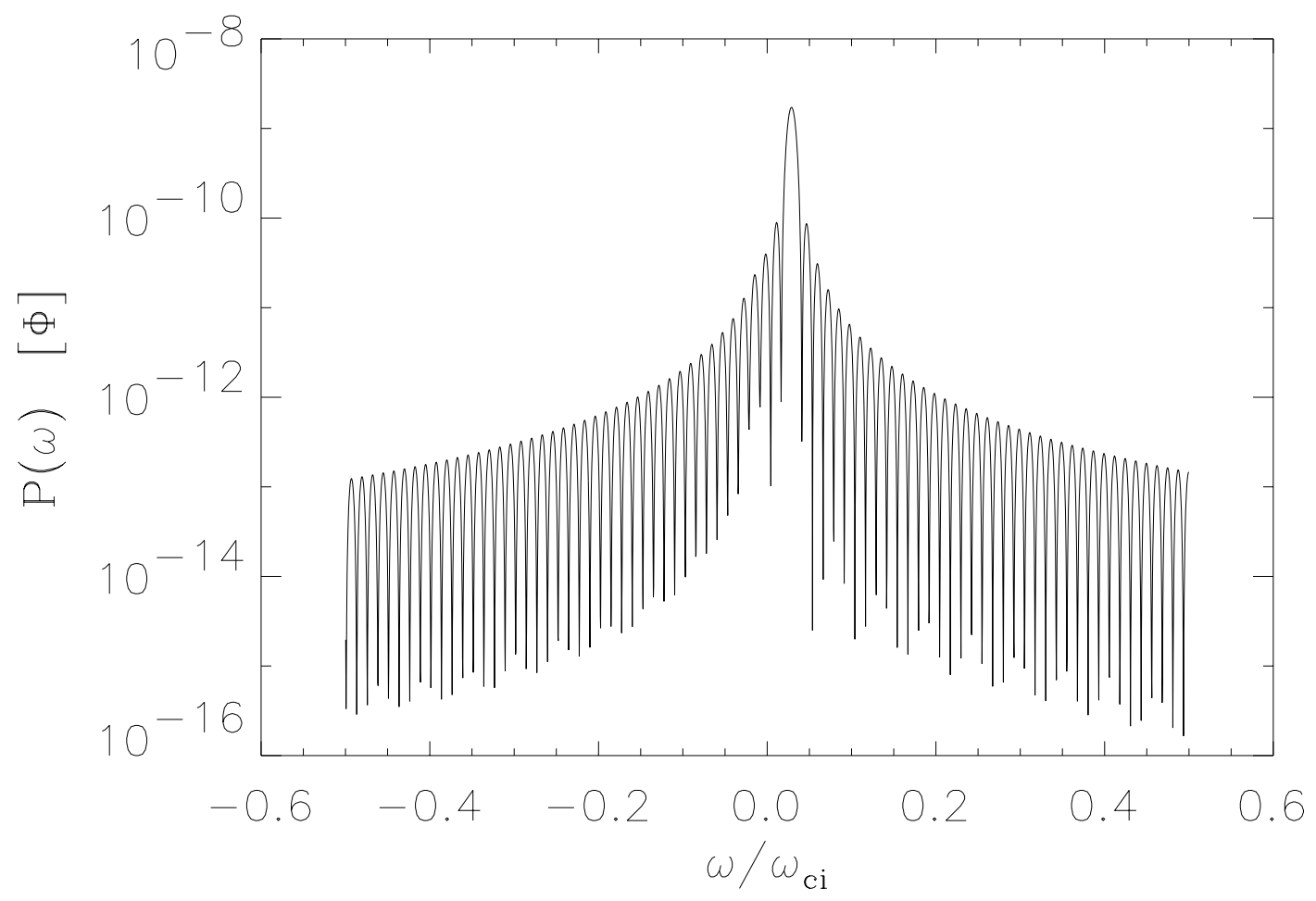




\section{FIG.11 Lewandowski}

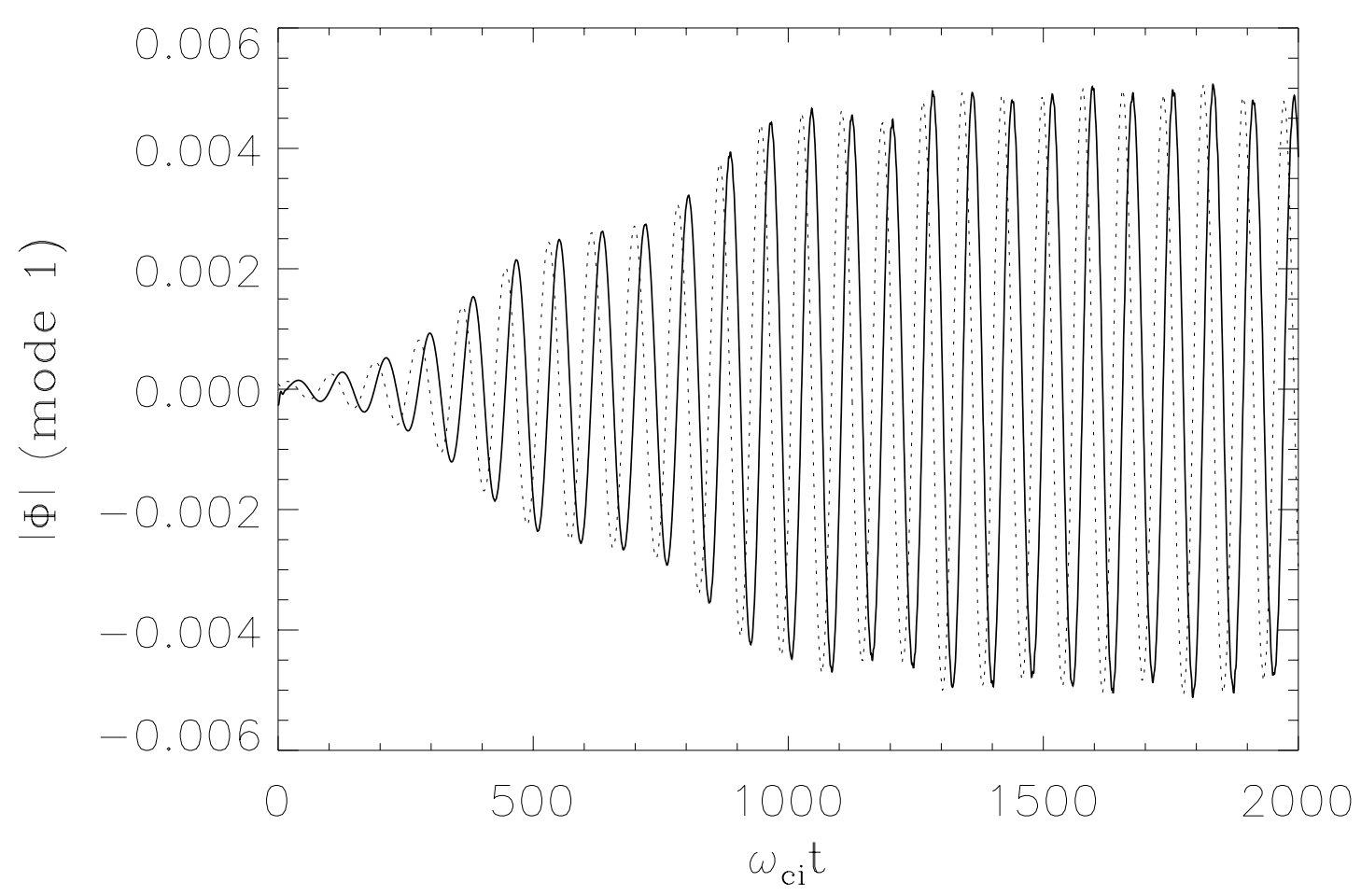




\section{FIG.12 Lewandowski}

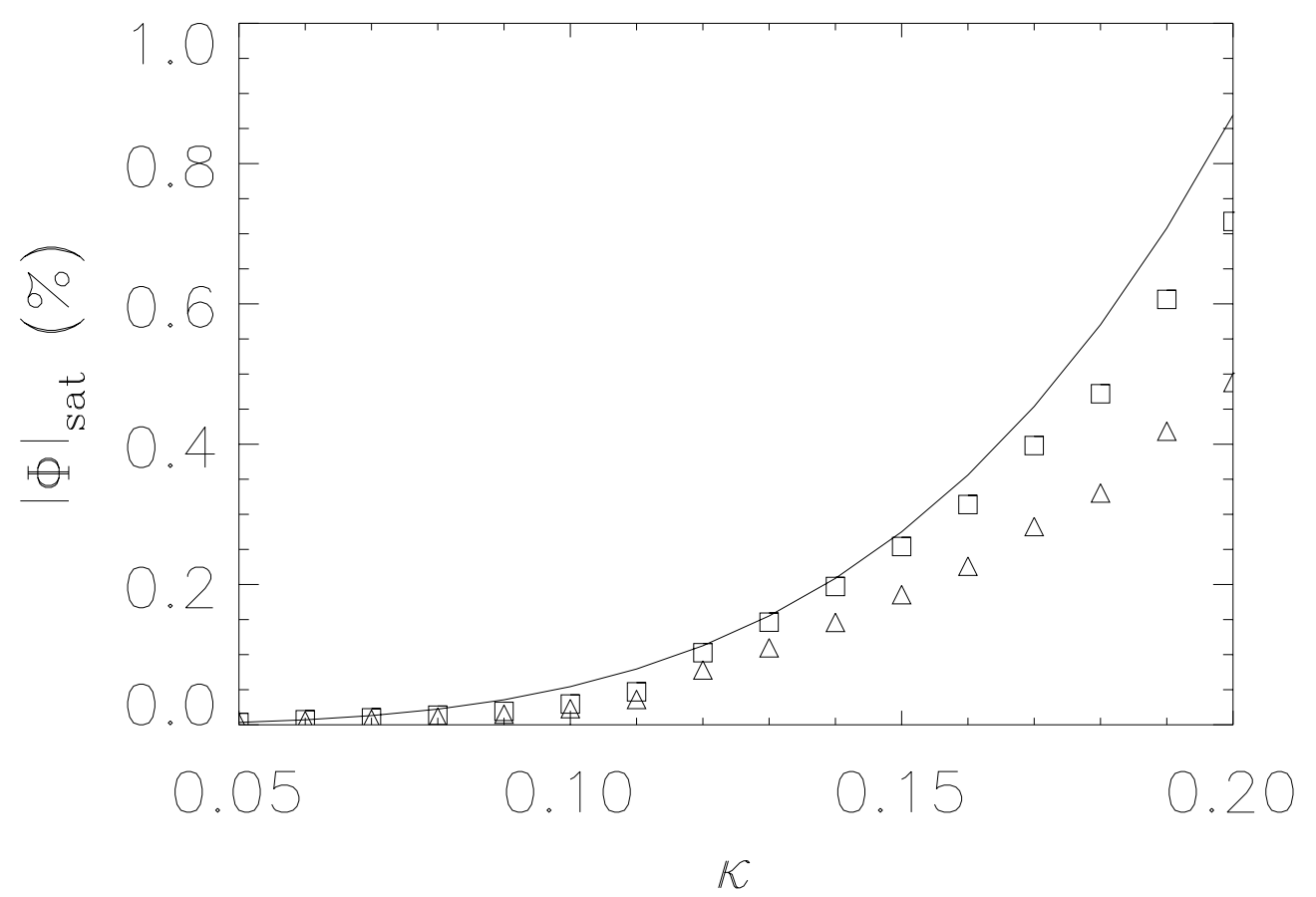




\section{External Distribution}

Plasma Research Laboratory, Australian National University, Australia

Professor I.R. Jones, Flinders University, Australia

Professor João Canalle, Instituto de Fisica DEQ/IF - UERJ, Brazil

Mr. Gerson O. Ludwig, Instituto Nacional de Pesquisas, Brazil

Dr. P.H. Sakanaka, Instituto Fisica, Brazil

The Librarian, Culham Laboratory, England

Mrs. S.A. Hutchinson, JET Library, England

Professor M.N. Bussac, Ecole Polytechnique, France

Librarian, Max-Planck-Institut für Plasmaphysik, Germany

Jolan Moldvai, Reports Library, MTA KFKI-ATKI, Hungary

Dr. P. Kaw, Institute for Plasma Research, India

Ms. P.J. Pathak, Librarian, Insitute for Plasma Research, India

Ms. Clelia De Palo, Associazione EURATOM-ENEA, Italy

Dr. G. Grosso, Instituto di Fisica del Plasma, Italy

Librarian, Naka Fusion Research Establishment, JAERI, Japan

Library, Plasma Physics Laboratory, Kyoto University, Japan

Research Information Center, National Institute for Fusion Science, Japan

Dr. O. Mitarai, Kyushu Tokai University, Japan

Dr. Jiangang Li, Institute of Plasma Physics, Chinese Academy of Sciences, People's Republic of China

Professor Yuping Huo, School of Physical Science and Technology, People's Republic of China

Library, Academia Sinica, Institute of Plasma Physics, People's Republic of China

Librarian, Institute of Physics, Chinese Academy of Sciences, People's Republic of China

Dr. S. Mirnov, TRINITI, Troitsk, Russian Federation, Russia

Dr. V.S. Strelkov, Kurchatov Institute, Russian Federation, Russia

Professor Peter Lukac, Katedra Fyziky Plazmy MFF UK, Mlynska dolina F-2, Komenskeho Univerzita, SK-842 15 Bratislava, Slovakia

Dr. G.S. Lee, Korea Basic Science Institute, South Korea

Institute for Plasma Research, University of Maryland, USA

Librarian, Fusion Energy Division, Oak Ridge National Laboratory, USA

Librarian, Institute of Fusion Studies, University of Texas, USA

Librarian, Magnetic Fusion Program, Lawrence Livermore National Laboratory, USA

Library, General Atomics, USA

Plasma Physics Group, Fusion Energy Research Program, University of California at San Diego, USA

Plasma Physics Library, Columbia University, USA

Alkesh Punjabi, Center for Fusion Research and Training, Hampton University, USA

Dr. W.M. Stacey, Fusion Research Center, Georgia Institute of Technology, USA

Dr. John Willis, U.S. Department of Energy, Office of Fusion Energy Sciences, USA

Mr. Paul H. Wright, Indianapolis, Indiana, USA 
The Princeton Plasma Physics Laboratory is operated by Princeton University under contract with the U.S. Department of Energy.

\author{
Information Services \\ Princeton Plasma Physics Laboratory \\ P.O. Box 451 \\ Princeton, NJ 08543
}

Phone: 609-243-2750

Fax: 609-243-2751

e-mail: pppl_info@pppl.gov

Internet Address: http://www.pppl.gov 